Supporting Information

for

\title{
Electrical and Thermal Transport through Silver Nanowires and Their Contacts - Effects of Elastic Stiffening
}

Yang Zhao, ${ }^{1}$ Matthew L. Fitzgerald, ${ }^{1}$ Yi Tao, ${ }^{1,2}$ Zhiliang Pan, ${ }^{1}$ Godfrey Sauti, ${ }^{3}$ Dongyan Xu, ${ }^{4}$

$$
\text { Ya-Qiong } \mathrm{Xu},{ }^{5,6} \text { and Deyu } \mathrm{Li}^{1, *}
$$

${ }^{1}$ Department of Mechanical Engineering, Vanderbilt University, Nashville, TN, 37235, USA

${ }^{2}$ School of Mechanical Engineering and Jiangsu Key Laboratory for Design and Manufacture of Micro-Nano Biomedical Instruments, Southeast University, Nanjing 210096, China

${ }^{3}$ NASA Langley Research Center, Hampton, VA 23681-2199

${ }^{4}$ Department of Mechanical and Automation Engineering, The Chinese University of Hong Kong, Shatin, New Territories, Hong Kong Special Administrative Region, China

${ }^{5}$ Department of Electrical Engineering and Computer Science, Vanderbilt University, Nashville, TN, 37235, USA

${ }^{6}$ Department of Physics and Astronomy, Vanderbilt University, Nashville, TN, 37235, USA

*: Author to whom correspondence should be addressed E-mail

E-mail: deyu.li@vanderbilt.edu 


\section{Contents}

I. Sample preparation and measurement method .......................................................... 3

II. Effects of the contact thermal resistance between nanowires and suspended membranes.... 3

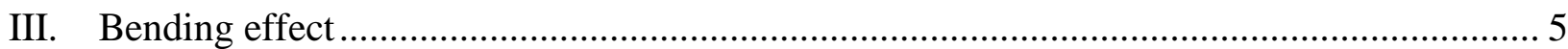

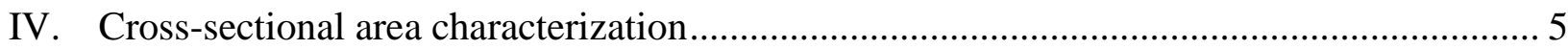

V. Cross-section cutting for contacted silver nanowires .................................................. 6

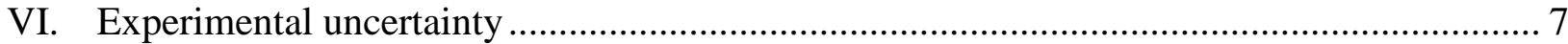

VII. Electrical resistivity fitting with the Bloch-Grüneisen (BG) formula .............................. 8

VIII. Effects of phonons on the Lorenz number ................................................................. 9

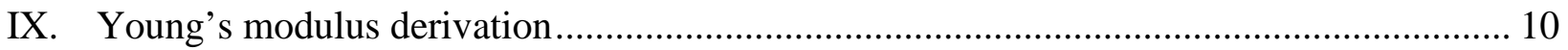




\section{Sample preparation and measurement method}

Silver nanowires suspended in isopropyl alcohol (IPA) with a concentration of $5 \mathrm{mg} / \mathrm{mL}$ were purchased from Sigma-Aldrich Corporation. A drop of IPA with silver nanowires was diluted with reagent alcohol and sonicated for 20 seconds to obtain a uniform dispersion. The obtained nanowire suspension was then casted onto a piece of polydimethylsiloxane (PDMS). After the solvent evaporated, an individual nanowire was picked up by a sharp probe mounted on an inhouse built micromanipulator, transferred to a measurement device and placed between two sideby-side suspended membranes. A small amount of reagent alcohol was dropped onto the membranes covering the parts of the nanowire, and the alcohol evaporation process helped to make an intimate contact between the nanowire and membranes to reduce the contact thermal resistance. ${ }^{1,2}$ Then, electron beam induced deposition (EBID) was employed to locally deposit Pt/C at the wire-suspended membrane contacts to further reduce the contact electrical and thermal resistance.

Thermal and electrical measurements were conducted with a microdevice in a cryostat (Janis CCS-400/204) under high vacuum $\left(<1 \times 10^{-6} \mathrm{mbar}\right)$ following a well-established approach that has been used to measure various types of one-dimensional (1D) nanostructures. ${ }^{3-6}$ The device consists of two suspended silicon nitride membranes with integrated platinum electrodes and resistance heaters/thermometers. The four electrodes enable four-point electrical conductance measurements. For the thermal conductance measurement, Joule heat generated by direct current flowing through the platinum resistor on the heating side can be transferred through the suspended silver nanowire to the sensing side and increase the membrane temperature. By measuring the total Joule heat generated at the heating side and temperature rises on both sides, the thermal conductance can be obtained. A Wheatstone bridge scheme was adopted for the sensing side to improve measurement sensitivity through rejecting the correlated temperature fluctuation in the cryostat. ${ }^{3}$ At each temperature point, we first measured the electrical resistance and then conducted thermal conductance measurement.

\section{Effects of the contact thermal resistance between nanowires and suspended membranes}

For metal nanowires with high electrical and thermal conductivities, the measurement results are very sensitive to the contact resistance between the nanowires and suspended membranes in our experimental scheme. For electrical measurements we adopted the four-point method so the effects of contact electrical resistance could be neglected; however, for thermal measurements the contact thermal resistance was still a concern even with EBID of Pt/C at the contacts. As shown in Fig. S1, even with Pt/C depositions, the contact thermal resistance of the silver nanowire with an outer-diameter of $125 \mathrm{~nm}$ could still lead to lower effective thermal conductivity, as evidenced by the inconsistent effective thermal conductance extracted before and after a second round of EBID. We note that this rather large contact thermal resistance could be due to that without EBID, the rather rigid large diameter Ag nanowires only make intimate contacts at several points with the not atomically smooth Pt electrodes. To minimize the effects from the contact thermal resistance, we wetted the contacts between the nanowire and suspended membranes with reagent alcohol, which, on evaporation, led to intimate contacts at the wire-membrane junctions. ${ }^{1,2}$ In addition, we prepared wire samples with a long-suspended segment between the two suspended membranes to increase the weight of the intrinsic wire resistance in the total measured value. 

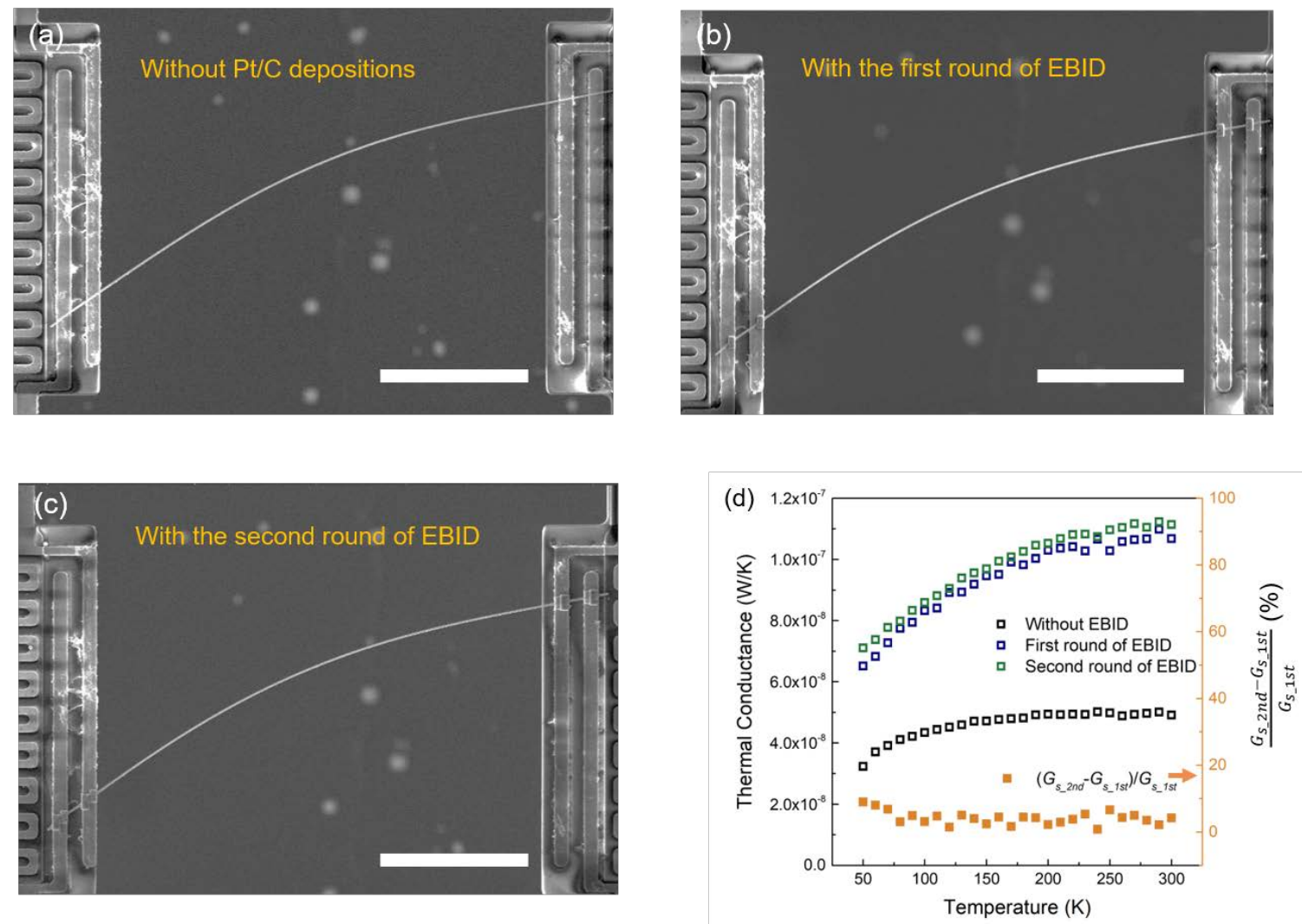

Figure S1. Thermal conductance of a silver nanowire with an outer-diameter of $125 \mathrm{~nm}$ and a suspended length of $33.7 \mu \mathrm{m}$. The scanning electron microscopy (SEM) images of the sample (a) without Pt/C deposition, (b) with the first and (c) with the second round of EBID of Pt/C at the wire-membrane contacts. (d) The measured thermal conductance increases significantly after the first round of EBID, and still increases slightly after the second round of EBID. The enhancement after the second round of EBID is within 10\%. The scale bar in (a), (b) and (c) is $10 \mu \mathrm{m}$.

Based on a fin model, ${ }^{7}$ the contact thermal resistance $\left(R_{C T R}\right)$ with each membrane can be expressed as

$$
R_{C T R}=\frac{1}{\sqrt{h P \kappa A} \tanh \left(L_{c m} \sqrt{\frac{h P}{\kappa A}}\right)},
$$

where $h$ is the heat transfer coefficient between the wire and membrane; $\kappa$ is the wire thermal conductivity; and $L_{c m}$ is the contact length which was kept approximately the same for all samples in our work. The fact that the second round of EBID does not alter the contact thermal resistance suggests that $R_{C T R}$ is independent of the contact length. In this case, $\tanh \left(L_{c m} \sqrt{\frac{h P}{\kappa A}}\right) \approx 1$, which also holds for smaller wires with lower $\kappa$ when $L_{c m}$ is unchanged. Thus, for nanowires with hydraulic diameter $D_{h}<89 \mathrm{~nm}, R_{C T R}=\frac{1}{\sqrt{h P \kappa A}}$. The ratio of $R_{C T R}$ to the intrinsic thermal resistance of the wire $\left(R_{i n}=L_{s} / \kappa A\right)$ is proportional to $\left(\kappa D_{h}\right)^{1 / 2} / L_{s}$, which is lower for smaller wires. 


\section{Bending effect}

After the thermal and electrical measurement on a nanowire sample, we used a sharp probe to create a kink defect at the middle of the nanowire and measured it again. The obtained thermal and electrical conductivities for the nanowire without and with the artificial kink defect essentially overlap with each other as shown in Fig. S2, which indicates that the effect of probe manipulation on the transport properties of the wire is negligible in our work.
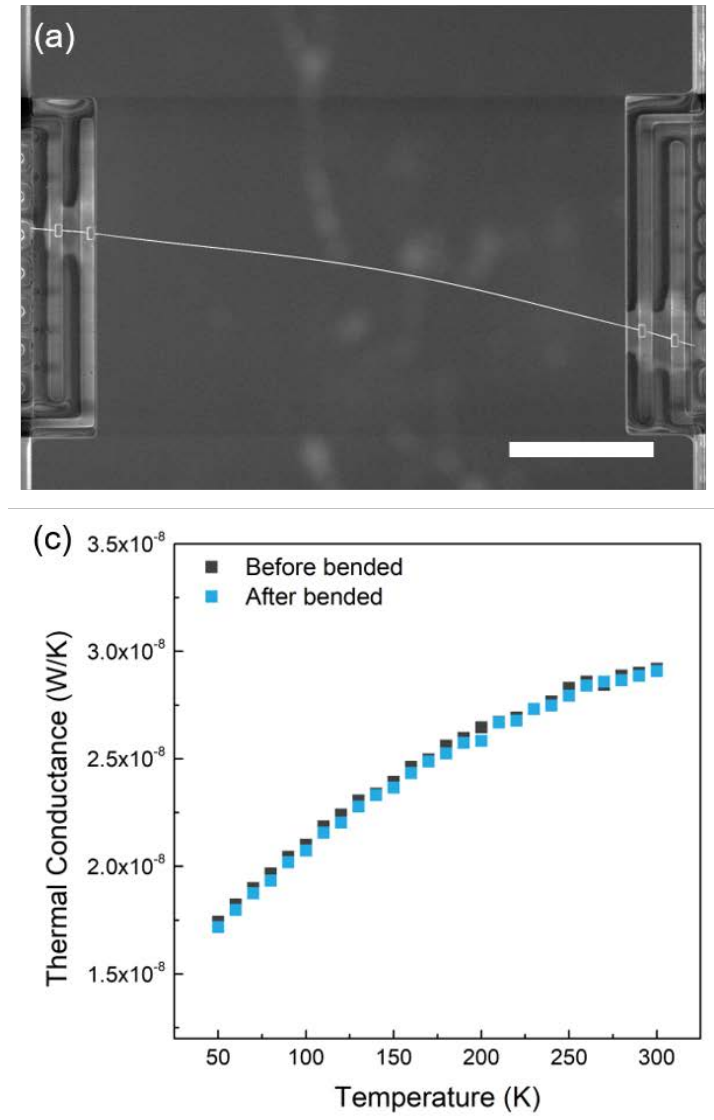
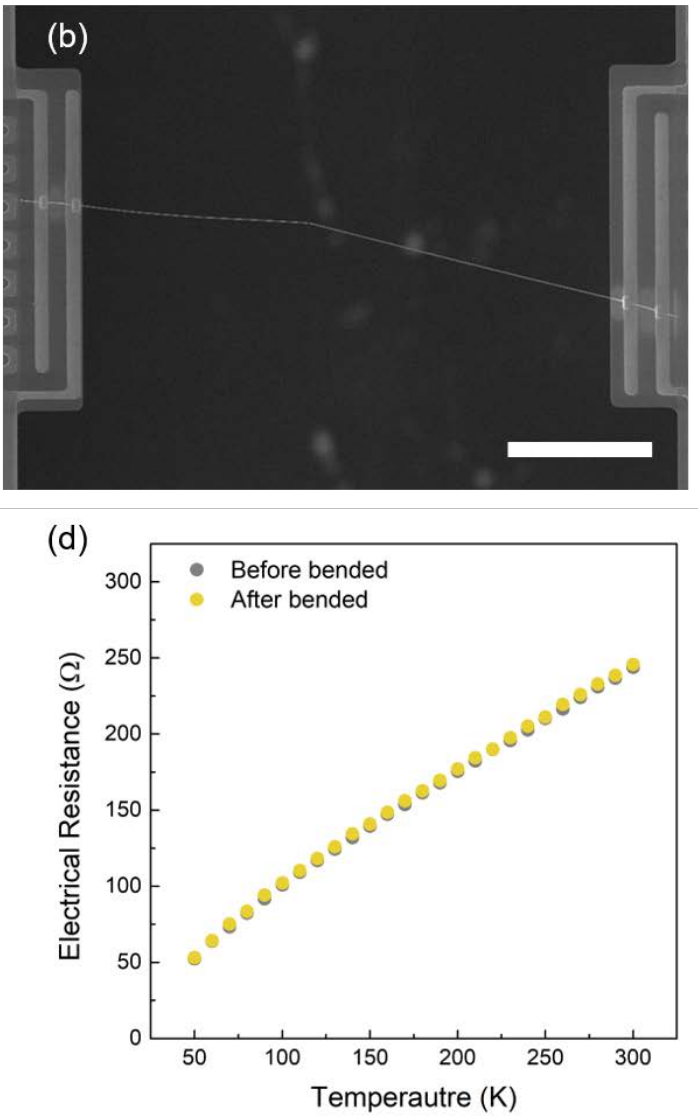

Figure S2. Examination of the effect of probe manipulation. A silver nanowire of 65 nm hydraulic diameter (a) without and (b) with an artificial kink defect. (c, d) Thermal conductance and electrical resistance before and after the kink is introduced. The scale bar in (a) and (b) is $10 \mu \mathrm{m}$.

\section{Cross-sectional area characterization}

The cross-sectional area was measured following the method used for $\mathrm{Ta}_{2} \mathrm{Pd}_{3} \mathrm{Se}_{8}$ and $\mathrm{NbSe}_{3}$ nanowires. $^{8,9}$ Fig. S3 shows the SEM micrographs (left panel) of the sample on the device and cross-sectional images (right panel) for the four silver nanowires reported in the main paper. 

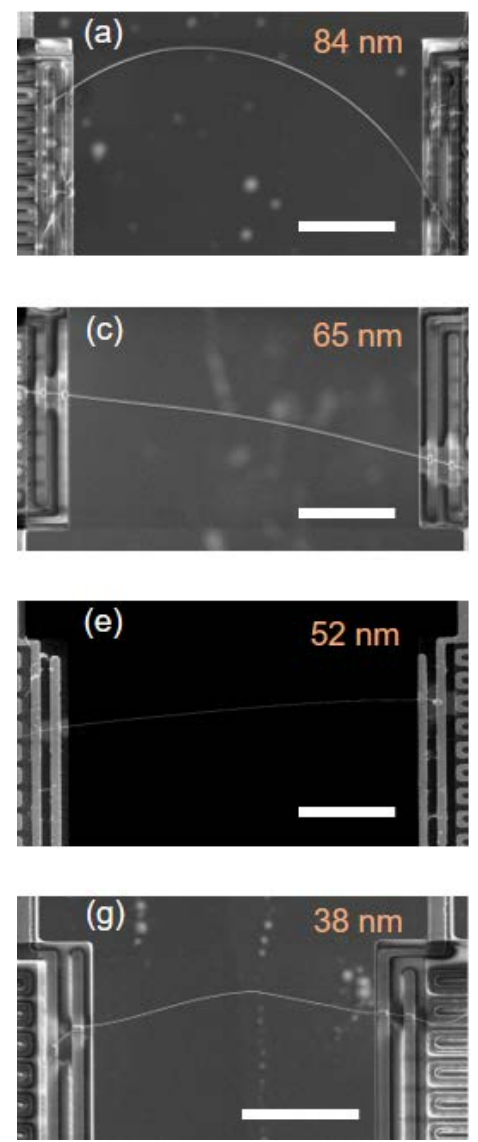
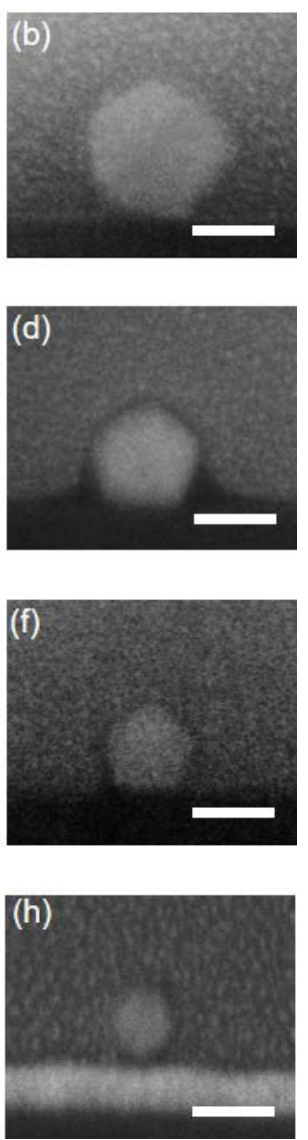

Figure S3. SEM images of measured samples on the device and cross-sectional view of the samples. The cross-section at the right side corresponds to the sample shown at the left side. The scale bar is $10 \mu \mathrm{m}$ for the left panels and $50 \mathrm{~nm}$ for the right panels.

\section{Cross-section cutting for contacted silver nanowires}

Fig. S4a shows the cross contact between two silver nanowires after the transport property measurement. EBID was done at the contact as shown in Fig. S4b. Then we transferred the contact to a silicon substrate for cross-section cutting with a focused ion beam. The process revealed the cross-sectional configuration, which was simultaneously examined by SEM with a tilted angle of $52^{\circ}$. Fig. S4c-f show the SEM images sequentially as the process proceeds to the junction of the two wires. 

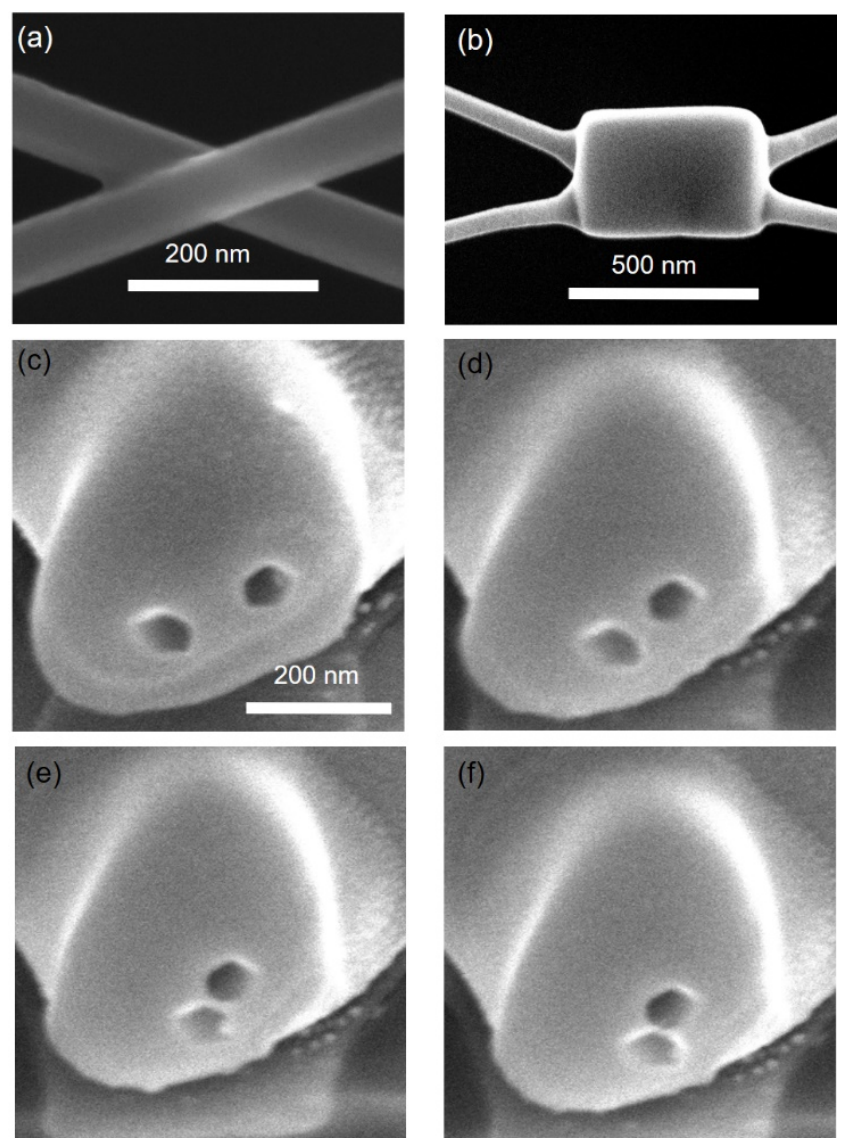

Figure S4. Cross-sectional images of the contact between two silver nanowires (65 nm hydraulic diameter). The scale bar for (c)-(f) is the same.

\section{Experimental uncertainty}

As the thermal and electrical resistivity were derived from the measured thermal conductance $(G)$ or electrical resistance $(R)$, suspended length and cross-sectional area $(A)$, based on the error propagation rule the relatively uncertainties for the thermal conductivity and electrical resistivity $(\rho)$ are derived as

$$
\begin{aligned}
& \frac{\delta \kappa}{\kappa}=\sqrt{\left(\frac{\delta G}{G}\right)^{2}+\left(\frac{\delta L_{S}}{L_{S}}\right)^{2}+\left(\frac{\delta A}{A}\right)^{2}}, \\
& \frac{\delta \rho}{\rho}=\sqrt{\left(\frac{\delta R}{R}\right)^{2}+\left(\frac{\delta L_{S}}{L_{S}}\right)^{2}+\left(\frac{\delta A}{A}\right)^{2}},
\end{aligned}
$$

The thermal conductance uncertainty mainly results from the electrical measurements, which is evaluated using a Monte Carlo method. In our measurement, $\delta G / G$ is $\sim 2 \%$ above $100 \mathrm{~K}$ and $~ 3 \%$ 
below $100 \mathrm{~K} .^{10,11}$ The electrical resistance uncertainty comes from the linear fitting of measured electrical voltage $(V)$ versus current $(I)$, which is calculated as

$$
U_{R}=\sqrt{\frac{\sum\left(V_{i}-R I_{i}-b\right)^{2}}{n-2} \times \frac{n}{\left(n \sum I_{i}^{2}\right)-\left(\sum I_{i}\right)^{2}}},
$$

where $b$ is the intercept and $n$ is the number of measured data. The relative uncertainty of electrical resistance becomes larger at low temperatures, which is $\sim 0.5 \%$ at $50 \mathrm{~K}$. The suspended length and cross-sectional area were characterized from SEM imaging, which gives rise to an uncertainty of $2 \mu \mathrm{m}$ in the length and a relatively uncertainty of $10 \%$ in the cross-sectional area. Based on Eq. S2 and S3, the overall uncertainty of thermal conductivity and electrical resistivity is $\sim 12 \%$ and $\sim 11 \%$ for measured silver nanowires, respectively.

\section{Electrical resistivity fitting with the Bloch-Grüneisen (BG) formula}

Fig. S5 shows the measured electrical resistivity of the nanowires with $D_{h}=84 \mathrm{~nm}$ and $38 \mathrm{~nm}$. The solid fitting line is derived based on BG formula as

$$
\begin{gathered}
\rho(T)=\rho_{0}+\rho_{e-p h}(T), \\
\rho_{e-p h}(T)=\alpha_{e-p h}\left(\frac{T}{\Theta_{D}}\right)^{5} \int_{0}^{\frac{\Theta_{D}}{T}} \frac{x^{5}}{\left(e^{x}-1\right)\left(1-e^{-x}\right)} d x,
\end{gathered}
$$

where $\rho_{0}$ is the residual resistivity due to defect scattering that is temperature-independent, $\rho_{e-p h}$ is the resistivity arising from electron phonon (e-ph) interactions, $\alpha_{e-p h}$ is a constant characterizing eph coupling, and $\Theta_{D}$ is the Debye temperature. ${ }^{12}$ The parameters used for the fitting is listed in the Table S1.

Table S1. The parameters used for the fitting line.

$\begin{array}{cccc}D_{h}(\mathbf{n m}) & \boldsymbol{\Theta}_{D}(\mathbf{K}) & \rho_{0}\left(\mathbf{1 0}^{-9} \boldsymbol{\Omega}-\mathbf{m}\right) & \boldsymbol{\alpha}_{\text {e-ph }}\left(\mathbf{1 0}^{-\mathbf{8}} \boldsymbol{\Omega}-\mathbf{m}\right) \\ \mathbf{8 4} & 128 & 1.67 & 3.47 \\ \mathbf{3 8} & 173 & 6.0 & 5.02\end{array}$




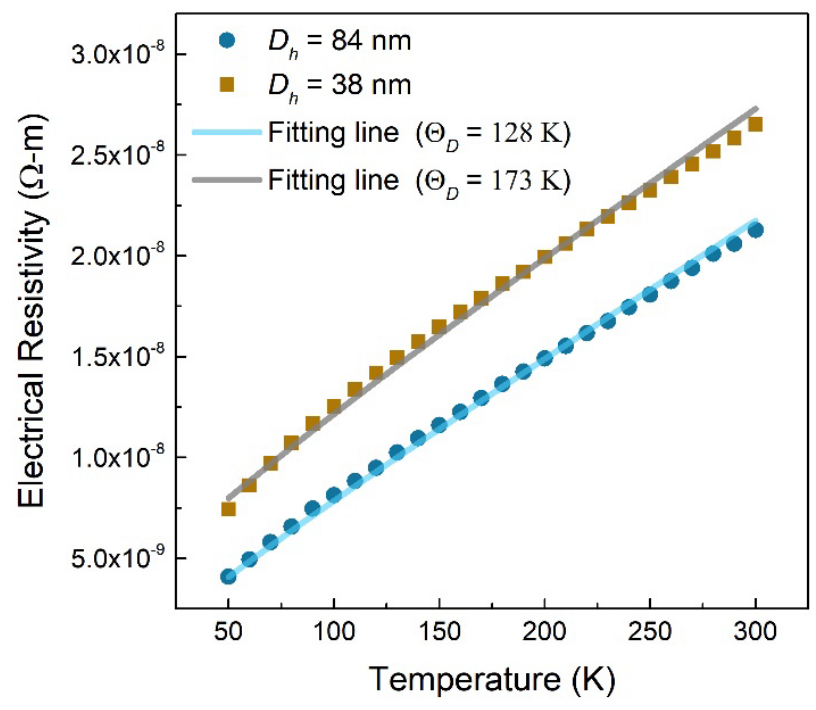

Figure S5. Electrical resistivity of the nanowires with $D_{h}=84 \mathrm{~nm}$ and $38 \mathrm{~nm}$.

\section{Effects of phonons on the Lorenz number}

As phonons still contribute a small portion to thermal transport in metals, the measured Lorenz number is expressed as

$$
L=\frac{\kappa_{p h}+\kappa_{e}}{\sigma T}
$$

where $\kappa_{p h}$ and $\kappa_{e}$ are the lattice and electronic thermal conductivity, respectively; $\sigma$ is the electrical conductivity; and $T$ is temperature. The phonon contribution to the overall thermal conductivity of silver predicted from a density functional theory calculation is $\sim 1.07 \%$ at $300 \mathrm{~K}^{13}$ Using this ratio the phonon thermal conductivity for bulk silver is $\sim 4.6 \mathrm{~W} / \mathrm{m}-\mathrm{K}$, which is assumed to be $\kappa_{p h}$ for the nanowires. By subtracting the lattice thermal conductivity, the Lorenz number purely due to electron transport $\left(L_{e}\right)$ can be derived as $\kappa_{e} / \sigma T$. As shown in Fig. S6, the calculated $L_{e}$, instead of being independent with size, increases as the wire diameter decreases, which suggests that phonon contribution without considering elastic stiffening cannot account for the Lorenz number enhancement in smaller wires. 


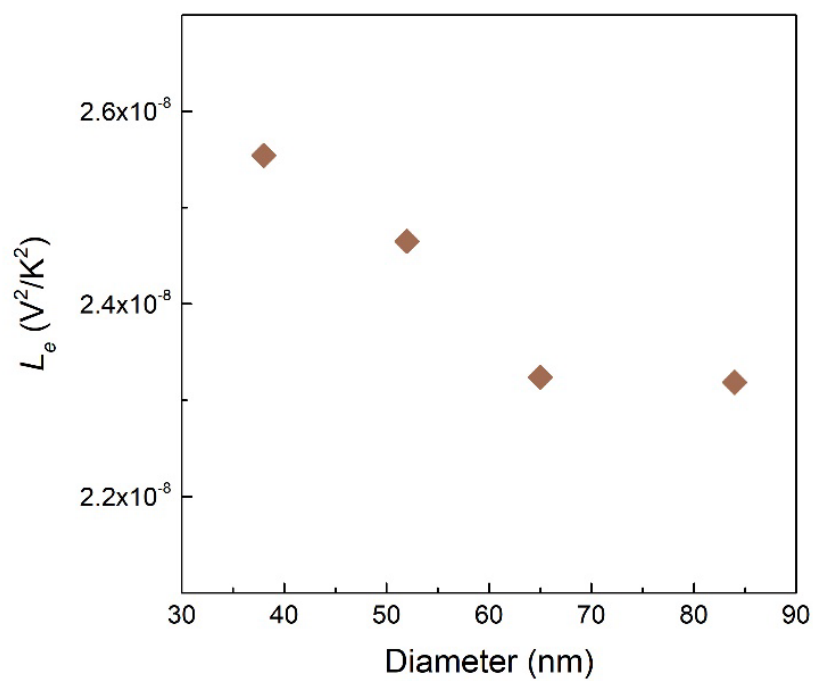

Figure S6. Calculated electronic Lorenz number at room temperature by subtracting the lattice thermal conductivity of bulk silver.

\section{Young's modulus derivation}

Fig. S7 shows the measured Young's modulus $(E)$ data for penta-twined silver nanowires with the third order polynomial fitting. The outer-diameter for the four nanowires with $D_{h}=84 \mathrm{~nm}, 65$ $\mathrm{nm}, 52 \mathrm{~nm}$ and $38 \mathrm{~nm}$ is $96 \mathrm{~nm}, 72 \mathrm{~nm}, 58 \mathrm{~nm}$ and $42 \mathrm{~nm}$, which corresponds to the fitted Young's modulus of $1,1.11,1.33$ and 1.71 times the bulk value, respectively. ${ }^{14}$

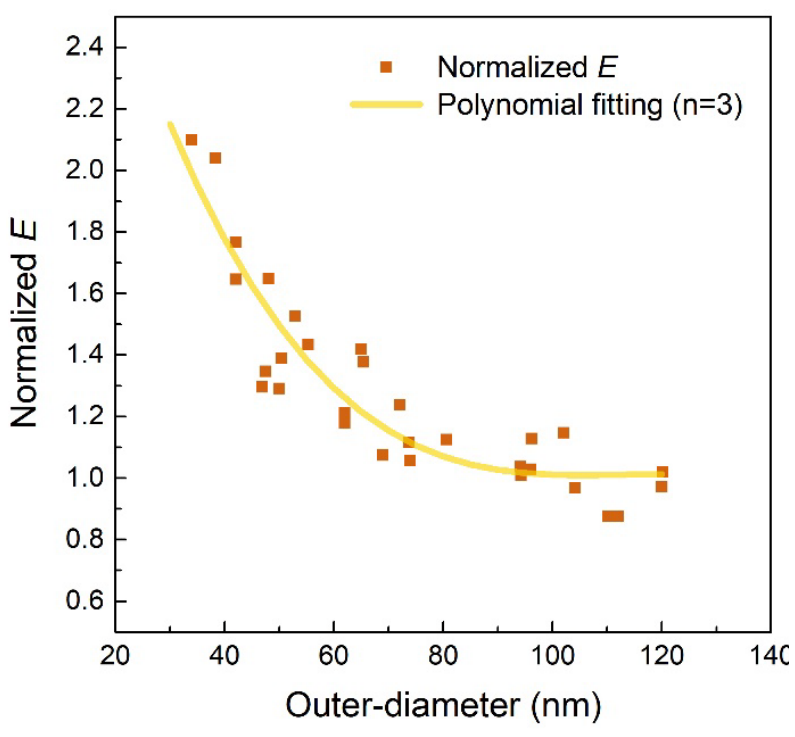

Figure S7. Extraction of the Young's modulus for the four nanowires with $D_{h}=84 \mathrm{~nm}, 65 \mathrm{~nm}$, $52 \mathrm{~nm}$ and $38 \mathrm{~nm}$, which correspond to outer diameters of $96 \mathrm{~nm}, 72 \mathrm{~nm}, 58 \mathrm{~nm}$ and $42 \mathrm{~nm}$, respectively. 


\section{Reference}

(1) Yang, J.; Yang, Y.; Waltermire, S. W.; Wu, X.; Zhang, H.; Gutu, T.; Jiang, Y.; Chen, Y.; Zinn, A. a; Prasher, R.; et al. Enhanced and Switchable Nanoscale Thermal Conduction due to van Der Waals Interfaces. Nature Nanotechnology 2012, 7, 91-95.

(2) Yang, L.; Zhang, Q.; Cui, Z.; Gerboth, M.; Zhao, Y.; Xu, T. T.; Walker, D. G.; Li, D. Ballistic Phonon Penetration Depth in Amorphous Silicon Dioxide. Nano Letters 2017, 17, 7218-7225.

(3) Wingert, M. C.; Chen, Z. C. Y.; Kwon, S.; Xiang, J.; Chen, R. Ultra-Sensitive Thermal Conductance Measurement of One-Dimensional Nanostructures Enhanced by Differential Bridge. Review of Scientific Instruments 2012, 83, 24901.

(4) Zhang, Q.; Cui, Z.; Wei, Z.; Chang, S. Y.; Yang, L.; Zhao, Y.; Yang, Y.; Guan, Z.; Jiang, Y.; Fowlkes, J.; et al. Defect Facilitated Phonon Transport through Kinks in Boron Carbide Nanowires. Nano Letters 2017, 17, 3550-3555.

(5) Yang, L.; Zhao, Y.; Zhang, Q.; Yang, J.; Li, D. Thermal Transport through Fishbone Silicon Nanoribbons: Unraveling the Role of Sharvin Resistance. Nanoscale 2019, 11, 8196-8203.

(6) Yang, L.; Zhang, Q.; Wei, Z.; Cui, Z.; Zhao, Y.; Xu, T. T.; Yang, J.; Li, D. Kink as a New Degree of Freedom to Tune the Thermal Conductivity of Si Nanoribbons. Journal of Applied Physics 2019, 126, 155103.

(7) Bifano, M. F. P.; Park, J.; Kaul, P. B.; Roy, A. K.; Prakash, V. Effects of Heat Treatment and Contact Resistance on the Thermal Conductivity of Individual Multiwalled Carbon Nanotubes Using a Wollaston Wire Thermal Probe. Journal of Applied Physics 2012, 111, 54321.

(8) Zhang, Q.; Liu, C.; Liu, X.; Liu, J.; Cui, Z.; Zhang, Y.; Yang, L.; Zhao, Y.; Xu, T. T.; Chen, Y.; et al. Thermal Transport in Quasi-1D van Der Waals Crystal Ta2Pd3Se8 Nanowires: Size and Length Dependence. ACS Nano 2018, 12, 2634-2642.

(9) Yang, L.; Tao, Y.; Liu, J.; Liu, C.; Zhang, Q.; Akter, M.; Zhao, Y.; Xu, T. T.; Xu, Y.; Mao, Z.; et al. Distinct Signatures of Electron-Phonon Coupling Observed in the Lattice Thermal Conductivity of NbSe3 Nanowires. Nano Letters 2019, 19, 415-421.

(10) Zhang, Q. Thermal Transport in Individual Nanowires of Fine Structures. Vanderbilt Thesis 2017, 549, 40-42.

(11) Yang, J.; Yang, Y.; Waltermire, S. W.; Gutu, T.; Zinn, A. A.; Xu, T. T.; Chen, Y.; Li, D. Measurement of the Intrinsic Thermal Conductivity of a Multiwalled Carbon Nanotube and Its Contact Thermal Resistance with the Substrate. Small 2011, 7, 2334-2340.

(12) Thermal Conductivity; Tritt, T. M., Ed.; Physics of Solids and Liquids; Springer US: New york, 2004.

(13) Jain, A.; McGaughey, A. J. H. Thermal Transport by Phonons and Electrons in Aluminum, Silver, and Gold from First Principles. Physical Review B 2016, 93, 81206.

(14) Chang, T.-H.; Cheng, G.; Li, C.; Zhu, Y. On the Size-Dependent Elasticity of PentaTwinned Silver Nanowires. Extreme Mechanics Letters 2016, 8, 177-183. 